\section{Cureus}

Received 08/06/2018

Review began 08/19/2018

Review ended 09/10/2018

Published 09/27/2018

\section{(c) Copyright 2018}

lqbal et al. This is an open access article distributed under the terms of the Creative Commons Attribution License CC-BY 3.0., which permits unrestricted use, distribution, and reproduction in any medium, provided the original author and source are credited.

\title{
Pseudogout: An Autoimmune Paraneoplastic Manifestation of Myelodysplastic Syndrome
}

\author{
Shumaila M. Iqbal ${ }^{1}$, Hafiz M. Aslam ${ }^{2}$, Faizan Faizee $^{3}$, Sana Qadir ${ }^{4}$, Saadia Waheed ${ }^{1}$ \\ 1. Internal Medicine, University at Buffalo / Sisters of Charity Hospital, Buffalo, USA 2. Internal \\ Medicine, Hackensack Meridian School of Medicine at Seton Hall University, Nutley, USA 3. Internal \\ Medicine, Dow University of Health Sciences (DUHS), Karachi, PAK 4. Internal Medicine, S \& A \\ Pediatrics, Parsippany, USA
}

$\square$ Corresponding author: Shumaila M. Iqbal, s.m.iqbal91@hotmail.com Disclosures can be found in Additional Information at the end of the article

\section{Abstract}

Myelodysplastic syndrome (MDS) is often associated with autoimmune paraneoplastic manifestations. Seronegative arthritis is among one of them. Very rarely, pseudogout demonstrated as paraneoplastic autoimmune manifestations of MDS has been adumbrated so far. Our case would be the another addition in the series. Our patient is an 83-year-old male lately diagnosed with MDS. After six months of initial diagnosis, he had a sudden onset episode of pain and swelling involving left wrist. Synovial fluid analysis from respective radiocarpal joint confirmed the presence of intracellular positively birefringent rhomboid shaped crystals of calcium pyrophosphate dihydrate (CPPD). This was followed by another two flares of pseudogout involving right knee and lumbar spine at separate time intervals. Each of the episodes mentioned above responded well to intravenous and oral steroids. After the third bout, he was started treatment with azacitidine which showed effective abatement of further episodes of pseudogout up until now.

Categories: Rheumatology

Keywords: key words: myelodysplastic syndrome, pseudogout, autoimmune, paraneoplastic

\section{Introduction}

The accumulation of calcium pyrophosphate dihydrate (CPPD) crystals in the articular tissues leads to numerous manifestations including asymptomatic chondrocalcinosis, chronic CPPD inflammatory arthritis and acute CPPD crystal arthritis (pseudogout) [1]. The risk factors associated with pseudogout include hyperparathyroidism, hypothyroidism, hemochromatosis, electrolyte imbalances such as hypomagnesemia and hypophosphatemia, osteoarthritis, old age and prior joint injury [2]. Hereditary traits are also infrequently associated with pseudogout. Myelodysplastic syndrome (MDS) has been associated with autoimmune paraneoplastic manifestations, such as vasculitis, glomerulonephritis, hypothyroidism, inflammatory bowel disease, peripheral neuropathies, and seronegative inflammatory arthritis [3]. Majority of them have demonstrated an effectual response to steroid therapy $[4,5]$. In the spectrum of seronegative inflammatory arthritides, as per authors' knowledge, pseudogout's association with MDS has been reported only a single time in the literature [1]. The reported case was found to be associated with complex karyotyping of MDS [1]. Discussed below is an anecdotal case of pseudogout manifesting as an autoimmune paraneoplastic sequela of MDS. 


\section{Cureus}

\section{Case Presentation}

An 83-year-old male patient presented to the hematology-oncology clinic with the past medical history of small bowel obstruction status post resection, basal cell carcinoma of the skin, actinic keratosis and colon adenocarcinoma status post left hemicolectomy with negative postoperative surveillance for relapse by serial carcinoembryonic antigen levels and serial colonoscopies. He developed progressing pancytopenia. His laboratory workup revealed, hemoglobin $9.1 \mathrm{~g} / \mathrm{dl}$, platelet count 76,000/ul, white blood cell (WBC) count 2700/ul with 59\% neutrophils, and absolute neutrophil count $1600 / \mathrm{mm}^{3}$. He had no reported past medical history of hematological disorders. No sign or symptom or any laboratory workup was indicative of systemic infection or inflammation. The patient's home medications did not include any antimetabolite nor he was ever treated in the past with any antineoplastic agents or radiation therapy. The patient was a lifetime nonsmoker with occasional alcohol drinking. Vitamin B12 and folic acid levels were normal and HIV tests were negative, respectively. Bone marrow aspiration was performed which revealed myelodysplasia with ring sideroblasts (Figures 1-3). Cytogenetic results delineated complex abnormal karyotype with monosomy of chromosomes $5,7,20$, partial deletion of $5 q$, and abnormalities consistent with high-grade myelodysplasia. The patient did not give consent for the proposed treatment with low dose chemotherapy. Thus management was begun with weekly intravenous (IV) erythropoietin administration along with as needed blood transfusion.

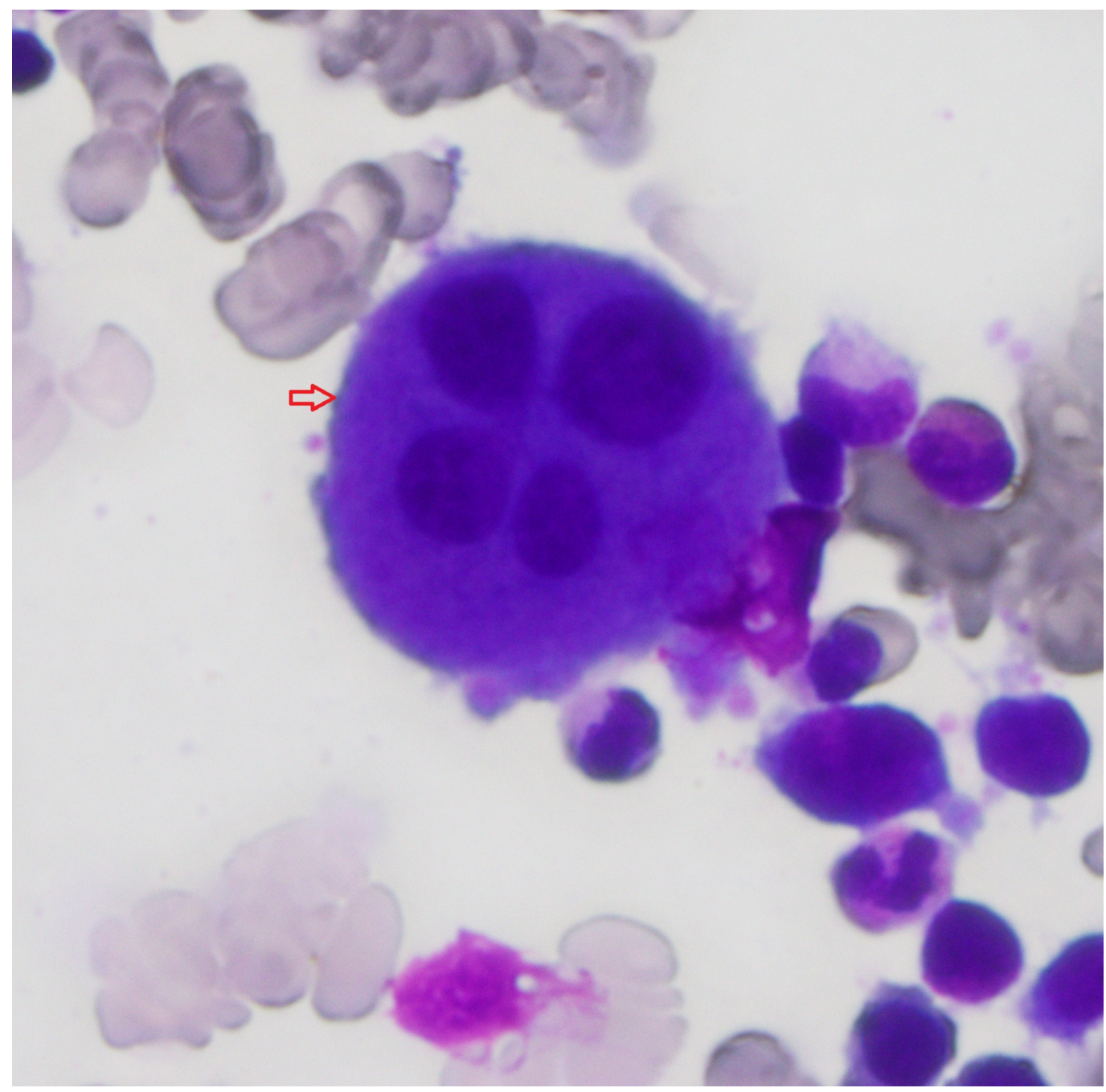

FIGURE 1: Bone marrow aspirant; arrow indicates dysplastic megakaryocyte. 


\section{Cureus}

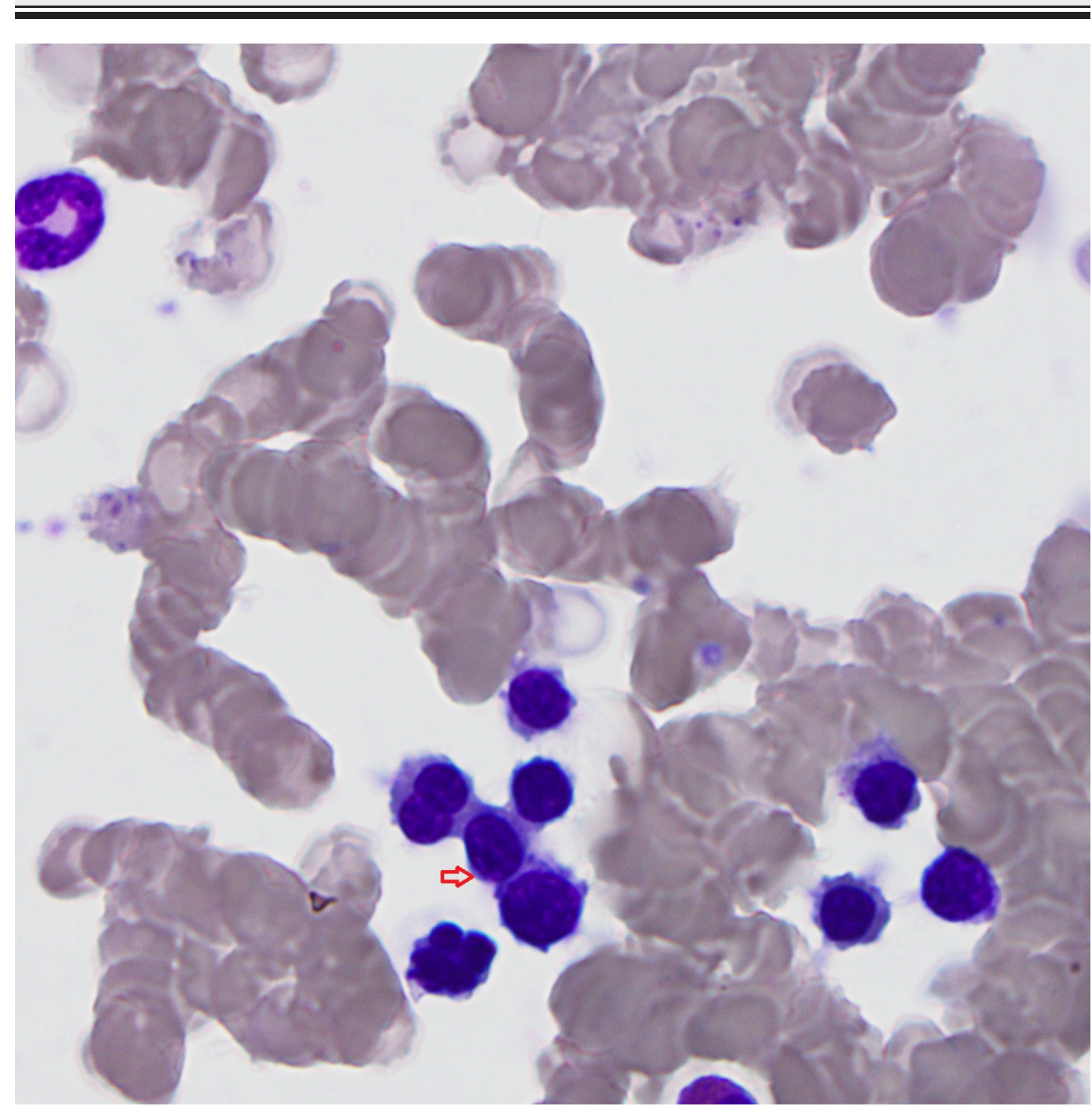

FIGURE 2: Bone marrow aspirant; arrow indicates dysplastic erythroids. 


\section{Cureus}

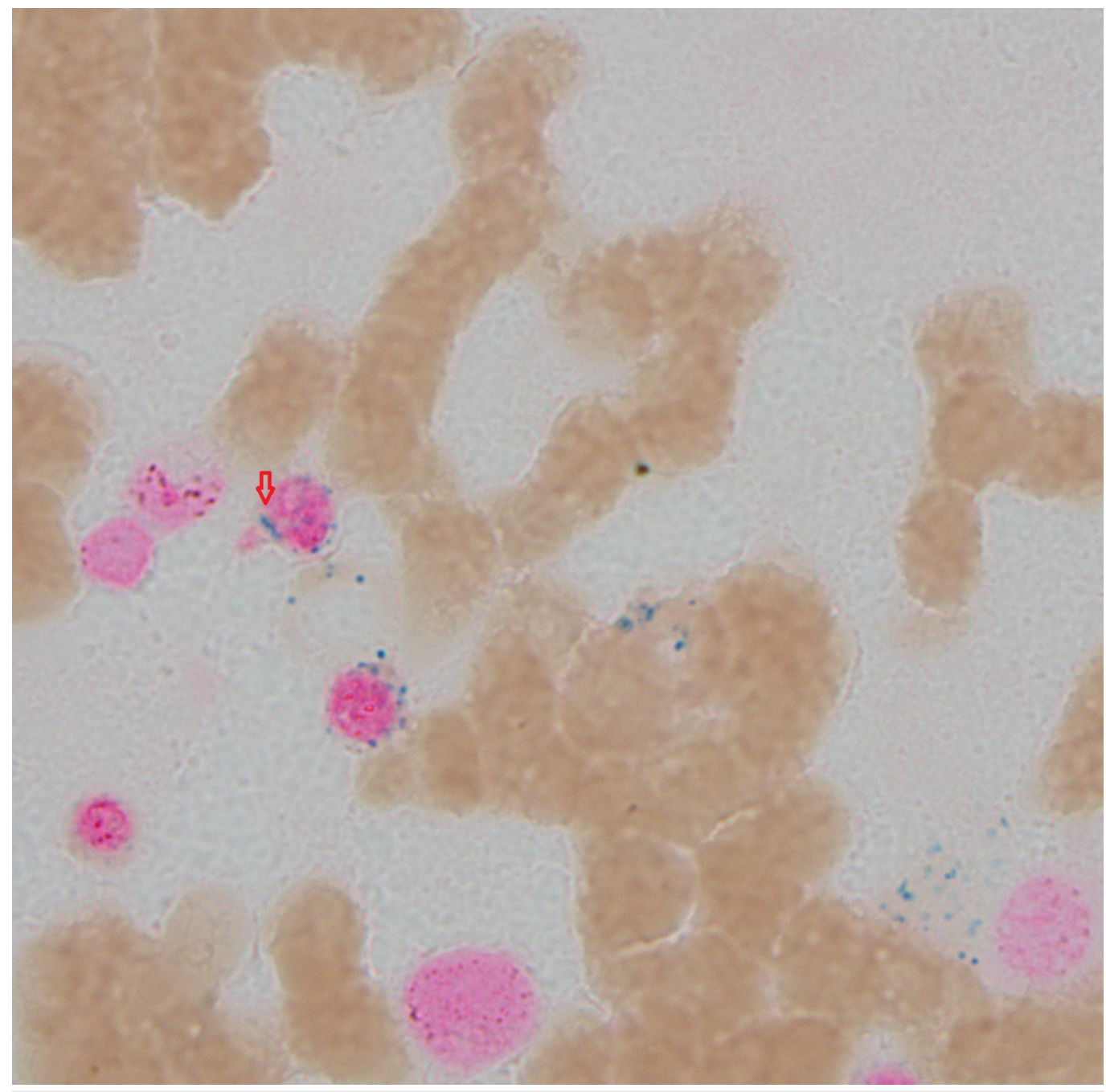

FIGURE 3: Bone marrow aspirant; arrow indicates ringed sideroblast which in turn is indicating dysplasia.

Six months post-diagnosis, the patient was presented to the emergency department with the chief complaint of pain, swelling and redness of the left wrist-joint. He was afebrile and physical examination exhibited a swollen joint which elicited pain upon movement. X-ray of the wrist was evident for chondrocalcinosis (Figure 4). Erythrocyte sedimentation rate (ESR) and C-reactive protein (CRP) levels were elevated. Lab workup revealed WBC count $1.8 \times 10^{9} / \mathrm{L}$ with $8 \%$ bands. Taking into account his leukopenia and inflamed wrist joint, the patient was empirically started on antibiotics for the clinical suspicion of septic arthritis but a minimal improvement in the symptoms was noticed. Synovial fluid extracted from the radiocarpal joint was grossly turbid with WBC count $6897 / \mathrm{mm}^{3}$ and polymorphonuclear granulocyte $87 \%$. Gram staining was unremarkable and fluid cultures were negative for microbial growth. Crystal analysis of synovial fluid demonstrated few positively birefringent rhomboid intracellular CPPD crystals. Treatment with IV steroid was begun which culminated in a dramatic improvement of symptoms. Antibiotics administration was sustained due to a possibility of coexisting septic arthritis in the clinical context of leukopenia. The patient was discharged home on a steroid taper and a short duration course of antibiotics. Post-discharge, outpatient workup remained negative for antinuclear antibody, rheumatoid factor and cyclic citrullinated peptide antibody. 


\section{Cureus}

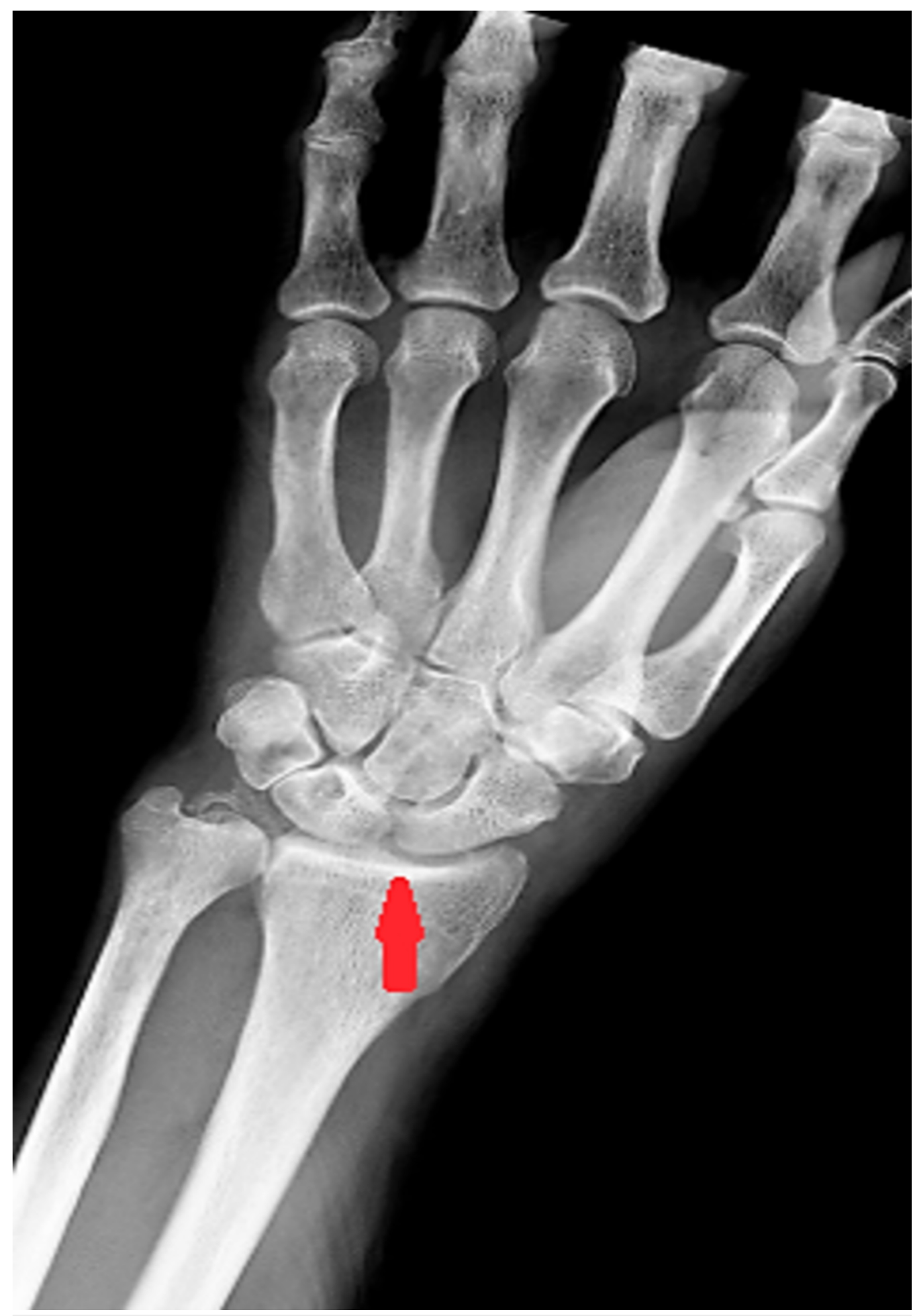

FIGURE 4: Chondrocalcinosis of radiocarpal joint.

A month later, the patient presented again with abrupt onset of right knee pain accompanied by joint swelling, tenderness and redness. X-ray indicated degenerative changes (chondrocalcinosis) of the knee joint most remarkable around the medial femorotibial compartment (Figure 5). Levels for ESR and CRP were elevated. Intracellular CPPD crystals were identified on synovial fluid analysis. The patient was treated with steroids and antibiotics (due to persistent leukopenia). The patient improves clinically. Colchicine could not be started for 


\section{Cureus}

prophylaxis of pseudogout due to persistent leukopenia.

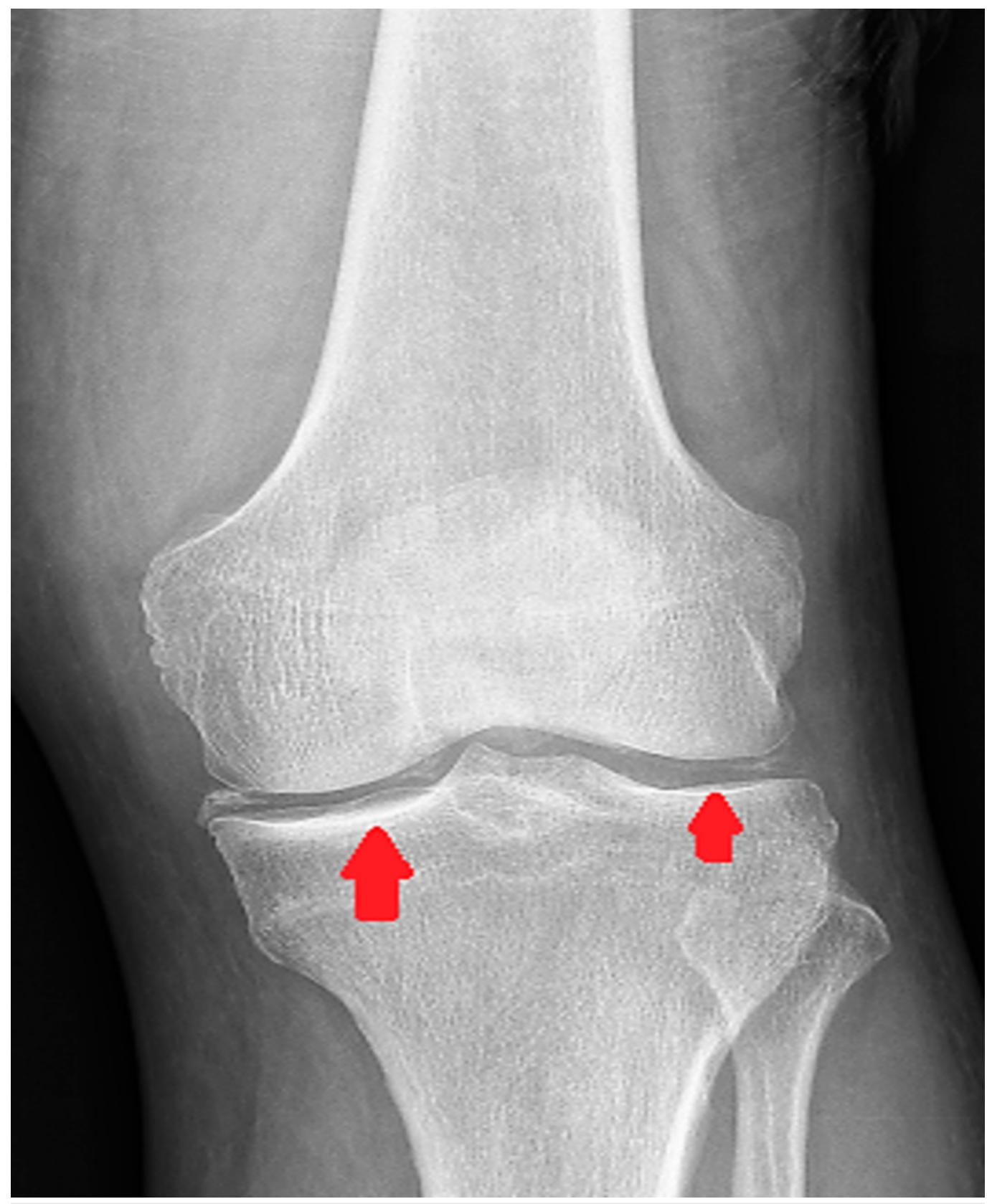

FIGURE 5: Chondrocalcinosis of knee joint.

A highly suspicious bout of pseudogout flared up again within a month with the symptoms of sharp lower back pain, localized to the lumbar area with redness surrounding overlying skin. The patient was afebrile. WBC count was 3500/ul. Degenerative changes were visible at L4-L5 on computed tomography (CT) spine with no contrast enhancing lesion identified on imaging (Figure 6). Considering history of previous flares of pseudogout, the patient was started treatment empirically with steroid in addition to antibiotics. Upon improvement, he was discharged home. Considering the evidence-based benefits of azacitidine in abeyance of the autoimmune phenomenon in MDS, the patient was then eventually started on azacitidine by his hematologist. No further acute CPPD crystal-associated acute flares of arthritis have been noted till date since the initiation of the therapy with azacitidine. 


\section{Cureus}

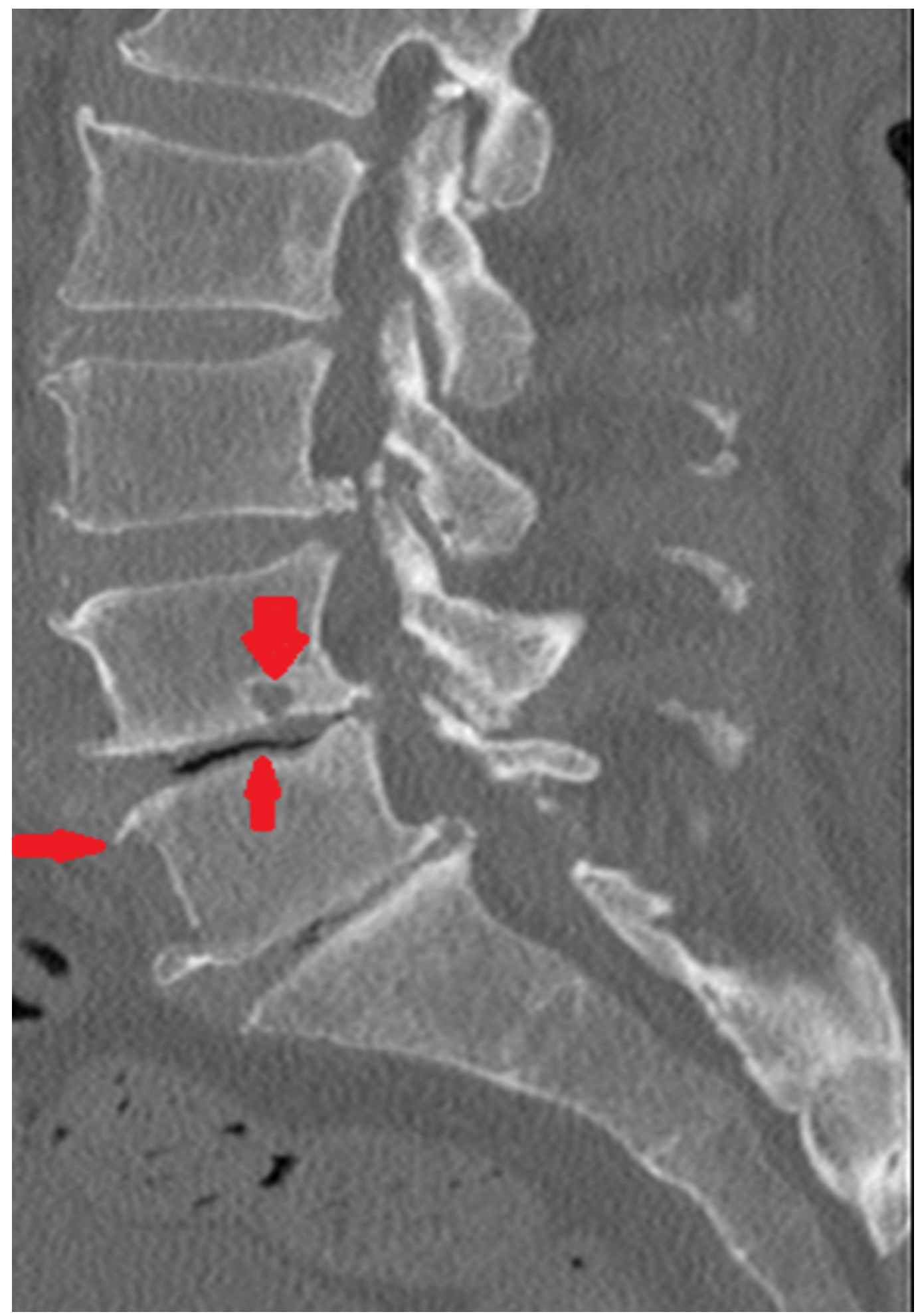

FIGURE 6: Degenerative changes at L4-L5.

\section{Discussion}

An intriguing case was reported in our hospital setting related to pseudogout associated with MDS. We found out a single case of this unique association in the medical literature [1]. Paraneoplastic syndrome is a term that describes a spectrum of clinical symptoms provoked by the production of different substances from a malignant neoplasm. The molecular mimicry portends by the released chemicals as well as the host immune response to the tumor cells 
results in various signs and symptoms [6]. The ubiquity of immune dysregulation in MDS characterized by an impaired CD8 response, an imbalance of T-regulatory cells and Th-17 cells also explain the inception of autoimmune manifestations [7]. At present, we do not have sufficient literature that could clarify the pathophysiological mechanisms behind the relationship of pseudogout with MDS. Hence, we hypothesize that our patient's seronegative inflammatory arthritis shares the same phenomenon of immune aberrancy and impaired T-cell response like the other autoimmune manifestations (AIMs) of MDS [8].

Our patient's arthritis did not convalesce with antibiotics as no organism was isolated from the synovial fluid tap; albeit, positively birefringent rhomboid intracellular calcium pyrophosphate crystals were detected in the synovial fluid which confirmed the diagnosis of pseudogout. Two causes, autoimmune paraneoplastic syndrome and administration of granulocyte colonystimulating factor (G-CSF) in MDS patients for neutropenia $[9,10]$ are reported to manifest pseudogout in MDS, the former being a renowned cause. Because our patient's multiple attacks of pseudogout involving several joints responded well to steroids, we speculated an autoimmune pathology behind it. Moreover, he was not administered G-CSF.

Cytogenetic abnormalities have been associated with AIM in MDS in nearly $50 \%$ of patients [11]. Numerous abnormal karyotypes have been recognised to provoke AIMs in MDS, however, 5-q deletions and monosomy 7 are the most commonly identified. Seronegative inflammatory arthritis, an AIM of MDS, is specifically found to be associated with more complex karyotyping [12]. The intricacy of cytogenetic abnormality is a prime tool in postulating the prognosis of AIM in MDS [11]. Deletions within the long arm (q) on chromosome 5 cause sustained NFkappa B signalling which results in activation of genes regulating inflammatory responses and apoptosis [13].

MDS-related arthritis involves multiple asymmetrical joints in a migratory fashion with infrequent association with positive Rh factor. Thus, autoimmune seronegative inflammatory arthritis is a classic type of arthritis observed in MDS patients [5, 7]. The precise mechanism of AIM in MDS remains elusive and requires extensive clinical data. Many clinical analyses have revealed that treating the intrinsic tumors can control both the progression of the tumor and its related AIMs [14]. Azacitidine and Decitabine have been documented to be beneficial in MDS; numerous evidence has conferred an exceptional response of bone marrow to treatment as well as remission of autoimmune symptoms [15]. Since we initiated our patient on azacitidine by taking into account its advantages, symptoms of autoimmunity or joints pain were reduced.

\section{Conclusions}

Myelodysplastic syndrome patients have been repeatedly reported with various AIM including seronegative inflammatory arthritis. Pseudogout should be suspected as one such possibility in context of seronegative inflammatory arthritis in patients with MDS. Since abnormal T-cell response is involved in the induction of autoimmune traits, steroid therapy serves as a cornerstone in subduing autoimmune flare of pseudogout in MDS. Moreover, MDS and its relevant paraneoplastic inflammation can adequately be rectified with Azacitidine. In this modern epoch of medicine, we foster the evolution of genuine therapeutic strategies for MDSassociated inflammatory arthritis.

\section{Additional Information Disclosures}

Human subjects: Consent was obtained by all participants in this study. Conflicts of interest: In compliance with the ICMJE uniform disclosure form, all authors declare the following:

Payment/services info: All authors have declared that no financial support was received from any organization for the submitted work. Financial relationships: All authors have declared 
that they have no financial relationships at present or within the previous three years with any organizations that might have an interest in the submitted work. Other relationships: All authors have declared that there are no other relationships or activities that could appear to have influenced the submitted work.

\section{Acknowledgements}

We wish to thank the patient and the clinical team who provided her care. We would also like to appreciate efforts of Dr. Basem Ahmed for providing us, the authors, with pathological slides of patient's bone marrow aspirate.

\section{References}

1. Tedeschi S, Stone R, Helfgott S: Calcium pyrophosphate crystal inflammatory arthritis (pseudogout) with myelodysplastic syndrome: a new paraneoplastic syndrome?. J Rheumatol. 2017, 44:1101-1102. 10.3899/jrheum.170106

2. Rho Y, Zhu Y, Zhang Y, Reginato A, Choi H: Risk factors for pseudogout in the general population. Rheumatology (Oxford). 2012, 51:2070-2074. 10.1093/rheumatology/kes204

3. Saif M, Hopkins J, Gore S: Autoimmune phenomena in patients with myelodysplastic syndromes and chronic myelomonocytic leukemia. Leuk Lymphoma. 2002, 43:2083-2092. 10.1080/1042819021000016186

4. Enright H, Miller W: Autoimmune phenomena in patients with myelodysplastic syndromes . Leuk Lymphoma. 1997, 24:483-489. 10.3109/10428199709055585

5. George S, Newman E: Seronegative inflammatory arthritis in the myelodysplastic syndromes . Semin Arthritis Rheum. 1992, 21:345-354. 10.1016/0049-0172(92)90035-c

6. Hirokawa M: Paraneoplastic autoimmune disorders (Article in Japanese). Gan To Kagaku Ryoho. 2010, 37:980-983.

7. Mekinian A, Braun T, Decaux O, et al.: Inflammatory arthritis in patients with myelodysplastic syndromes: a multicenter retrospective study and literature review of 68 cases. Medicine (Baltimore). 2014, 93:1-10. 10.1097/md.0000000000000011

8. Wolach O, Stone R: Autoimmunity and inflammation in myelodysplastic syndromes . Acta Haematol. 2016, 136:108-117. 10.1159/000446062

9. Ames P, Rainey M: Consecutive pseudogout attacks after repetitive granulocyte colonystimulating factor administration for neutropenia. Mod Rheumatol. 2007, 17:445-446. 10.1007/s10165-007-0601-z

10. Yamamoto H, Teramoto S, Matsui H, Matsuse T, Toba K, Ouchi Y: An elderly case with pseudogout exacerbated by the administration of granulocyte-colony stimulating factor during drug-induced granulocytopenia (Article in Japanese). Nihon Ronen Igakkai Zasshi. 1999, 36:572-575.

11. Haase D: Cytogenetic features in myelodysplastic syndromes . Ann Hematol. 2008, 87:515-526. 10.1007/s00277-008-0483-y

12. Mekinian A, Grignano E, Braun T, et al.: Systemic inflammatory and autoimmune manifestations associated with myelodysplastic syndromes and chronic myelomonocytic leukaemia: a French multicentre retrospective study. Rheumatology (Oxford). 2016, 55:291300. 10.1093/rheumatology/kev294

13. Fang J, Barker B, Bolanos L, et al.: Myeloid malignancies with chromosome $5 \mathrm{q}$ deletions acquire a dependency on an intrachromosomal NF-KB gene network. Cell Rep. 2014, 8:13281338. 10.1016/j.celrep.2014.07.062

14. Marengo M, Suarez-Almazor M, Lu H: Neoplastic and paraneoplastic synovitis. Rheum Dis Clin North Am. 2011, 37:551-572. 10.1016/j.rdc.2011.09.008

15. Al Ustwani O, Francis J, Wallace P, Ambrus J, Wetzler M: Treating myelodysplastic syndrome improves an accompanying autoimmune disease along with a reduction in regulatory T-cells. Leuk Res. 2011, 35:35-36. 10.1016/j.leukres.2010.12.015 